\title{
RoboFácil: Especificação e Implemen- tação de um Kit de Robótica para a Realidade Educacional Brasileira
}

\author{
Leonardo Cunha de Miranda \\ Instituto de Computação \\ Universidade Estadual de Campinas \\ 13083-852, Campinas, SP, Brasil \\ professor@leonardocunha.com.br
}

\author{
Fábio Ferrentini Sampaio \\ Programa de Pós-Graduação em Informática \\ Núcleo de Computação Eletrônica \\ Universidade Federal do Rio de Janeiro \\ 20001-970, Rio de Janeiro, RJ, Brasil \\ ffs@nce.ufrj.br
}

\author{
José Antonio dos Santos Borges \\ Núcleo de Computação Eletrônica \\ Universidade Federal do Rio de Janeiro \\ 20001-970, Rio de Janeiro, RJ, Brasil \\ antonio2@nce.ufrj.br
}

Resumo Este artigo apresenta as tecnologias associadas a um kit de robótica de baixo custo com fins
educacionais desenvolvido com o intuito de promover um maior acesso a esse tipo de recurso
didático nas escolas brasileiras. Para alcançarmos esse objetivo, primeiramente realizamos
estudos comparativos entre os diferentes kits comercializados no mercado, focados nos custos e
nos recursos de hardware e software desses produtos. Baseado nesse levantamento, que nos
proporcionou um maior entendimento das necessidades de projeto e do seu aproveitamento em
sala de aula, chegamos a especificação e a implementação de hardware e de software do kit de
robótica educacional denominado RoboFácil.

Palavras-Chave: Robótica Educacional, Hardware e Software, Informática na Educação

\begin{abstract}
This paper presents the technologies associated with a low cost educational robotics kit developed with the intent to promote access to this kind of educational technology in Brazilian schools. To reach our goal, we first conducted comparative studies with different kits commercialized in the market, mainly focusing on the costs and features of the hardware and software. These studies gave us a better understanding of project requirements and the employment during class, and led to the specification and implementation of hardware and software of the educational robotics kit called RoboFácil.
\end{abstract}

Keywords: Educational Robotics, Hardware and Software, Computers in Education 


\section{Introdução}

Há algumas décadas diferentes teóricos vêm propondo e discutindo a inserção das Tecnologias da Informação e da Comunicação (TICs) no ambiente educacional. Busca-se com o emprego dessas tecnologias na Educação, uma melhor qualidade do ensino e ambientes de aprendizagem mais ricos e motivadores para os discentes.

Dentre o amplo espectro de ideias e propostas, no que se refere aos artefatos computacionais, é notório observar que as soluções exploram, em sua maioria, apenas a vertente de software. No entanto, a demanda por novos aparatos de hardware na Educação vem crescendo sendo evidenciada, sobretudo, pelos esforços da comunidade acadêmica em propor a inserção da robótica com fins pedagógicos, apoiados em resultados favoráveis à sua aplicação como, por exemplo, os descritos em [1,3,6,7,14,16,17,18,20]. Em relação a esses trabalhos, vale destacar que a maioria fez uso de kits de robótica importados. De fato, poucos são os trabalhos publicados que apresentam alternativas de kits para o contexto nacional $[4,5,15]$.

A robótica educacional é uma atividade desafiadora e lúdica, que utiliza o esforço do educando na criação de soluções de hardware e software visando a resolução de uma situação-problema proposto. Alguns projetos pedagógicos de robótica em sala de aula fazem uso, por exemplo, da teoria construtivista de Jean Piaget para auxiliar o processo de ensino permitindo ao aluno, no processo de construção do conhecimento, a oportunidade de participar de uma aprendizagem mais efetiva e desenvolver uma percepção mais acurada dos fenômenos científicos.

Para Schons et al. ([16], p. 5), a robótica pedagógica "constitui nova ferramenta que se encontra à disposição do professor, por meio da qual é possível demonstrar na prática muitos dos conceitos teóricos, às vezes de difícil compreensão, motivando tanto o professor como principalmente o aluno". Segundo Zilli [20], a robótica educacional pode desenvolver as seguintes competências: raciocínio lógico; formulação e teste de hipóteses; relações interpessoais; investigação e compreensão; representação e comunicação; resolução de problemas por meio de erros e acertos; aplicação das teorias formuladas a atividades concretas; criatividade; e capacidade crítica.

Podemos citar algumas vantagens com a adoção de kits de robótica educacional existentes no mercado: i) hardware e software construído direcionado a atender propósitos educacionais específicos; ii) flexibilidade na forma de utilização em diferentes aplicações; iii) existência de documentação técnica e manual do usuário dos produtos incluindo, em alguns casos, material pedagógico de apoio às atividades do professor em sala de aula; e iv) maior facilidade para aquisição e operação por usuários não familiarizados/especializados com as tecnologias de eletrônica ou informática.

Poucos são os produtos que disponíveis para comercialização no Brasil possuem uma boa relação entre recursos e custos. $\mathrm{O}$ acesso e o uso efetivo desses kits no Brasil é dificultado pelo fato dos modelos importados terem, normalmente, um elevado custo para os padrões nacionais e os fabricados no Brasil possuírem limitações de hardware ou software para algumas aplicações como, por exemplo, a impossibilidade do hardware permanecer desconectado do computador durante sua execução, ou de não permitir a reprogramação de seu comportamento via uma linguagem de programação visual.

Entendemos que a geração de soluções alternativas para resolver os problemas mencionados podem ajudar na promoção de um maior emprego dessa ferramenta pedagógica nas escolas brasileiras com poucos recursos econômicos, principalmente pelo público-alvo desta pesquisa, ou seja, os estudantes adolescentes do Ensino Médio. Nesse contexto, apresentamos neste artigo o kit de robótica educacional RoboFácil, que é um produto resultante de um projeto de pesquisa [11] realizado com o objetivo de criar soluções alternativas aos problemas supramencionados.

Este artigo está organizado da seguinte maneira: a Seção 2 descreve as tecnologias de hardware e software que foram desenvolvidas nesta pesquisa; na Seção 3 realizamos uma discussão sobre as tecnologias apresentadas neste artigo; e na Seção 4 tecemos nossas considerações finais e indicados trabalhos que poderão promover a continuidade desta pesquisa.

\section{Kit RoboFácil}

O RoboFácil é um kit de robótica com fins pedagógicos composto por diferentes elementos de hardware e software, conforme descrevemos a seguir.

\subsection{Hardware RoboFácil}

O hardware RoboFácil é baseado em uma CPU da família dos microcontroladores MCS-51 da Intel e contém uma extensão de 16 portas digitais de entrada e 16 portas de saída com buffer.

O sistema provê a utilização de circuitos externos plugins eletrônicos ou, simplesmente, plugins - que permitem a interação do sistema com o mundo externo. Um plugin acoplado ao hardware pode assim controlar, por exemplo, motores de passo, sensores de luminosidade e 
temperatura etc. De forma geral, os principais elementos eletrônicos que compõem este hardware são:

- CPU Intel 8031AH (CMOS de baixo consumo);

- $32 \mathrm{~KB}$ de ROM de programa e $32 \mathrm{~KB}$ de RAM de programa;

- 8 KB de RAM de dados;

- Interface de comunicação RS-232C;

- Display de 1 linha com 16 colunas;

- Teclado com 5 botões;

- Controle de motores de passo;

- Controle de motores DC;

- Sensor de temperatura;

- Sensor de luminosidade;

- Conversor digital-analógico;

- Conversor analógico-digital.

\subsubsection{Unidade de Controle e Sistema de Memória}

As características da unidade de controle derivam das propriedades operacionais das CPUs da linha Intel 8051, já projetada visando a implementação de sistemas de controle de equipamentos por microprocessador fazendo uso de um hardware mínimo. Existem diversos modelos dessas CPUs, cada um podendo ser configurado de diferentes formas.

A CPU 8051 é composta por três conjuntos de portas externas bidirecionais de 8 bits endereçáveis bit a bit (P0, P1 e P2). Alguns modelos dessa CPU podem ser utilizados com ROM e RAM externas e, nesse caso, duas portas (P0 e P2) são empregadas para controle dos barramentos externos de dados e endereços. Ao implementar o barramento as portas P0 e P2 são concatenadas para formar um endereço de 16 bits, dando assim o máximo de $2^{16}=64$ KBytes de acesso externo. A porta P0 é multiplexada através de um circuito 74HCT373 para fornecer o barramento bidirecional de dados.

A CPU implementa internamente um serializador/desserializador que serve para realizar a conexão serial do processador com o mundo externo. É possível configurá-lo para comunicação síncrona e assíncrona, embora tenhamos utilizado apenas a forma assíncrona.

A CPU possui sinais diferentes para leitura de programa e leitura de dados, aumentando teoricamente a quantidade possível de memória. Esse fato foi aproveitado neste projeto, servindo-se de uma área de ROM para rotinas básicas (32 KB), uma área de RAM para programa (32 KB) e uma área de RAM para dados (8 KB). Dessa forma, simplificamos o processo de testagem de novas rotinas básicas carregando-as, via interface serial, na área de RAM de programa. Cabe comentar que o bootstrap é feito a partir das rotinas já existentes em ROM.

Devido ao grande número de portas utilizadas neste projeto, foi escolhido o modelo de endereçamento de portas no espaço em memória. Assim, para os programas as diversas interfaces externas são lidas e gravadas como posições de memória. Dois circuitos NAND (74HCT00) e dois registradores de 4 bits (74HCT139) controlam e selecionam esses endereços e suas incompatibilidades com o espaço real de memória.

Apresentamos na Figura 1 o esquema do circuito eletrônico principal do RoboFácil. Os esquemas dos demais circuitos deste hardware estão disponíveis em [11].

\subsubsection{Comunicação}

Os sinais de comunicação serial da CPU são TXD e RXD (Transmitter e Receiver Data), que são ligados ao exterior através de uma conversão de nível e corrente, realizada por um circuito MC145407. Esse circuito permite a interconexão de seis sinais que podem ser aproveitados para gerar quatro outros sinais viabilizando, dessa forma, o controle de um modem externo e permitindo, por exemplo, a sua conexão à Internet. Esses sinais extras são lidos numa porta específica a partir de um latch 74HCT244.

Os sinais gerados por esse circuito são levados a um conector externo (DB-25F) preparado para conexão a um cabo serial que, por sua vez, é conectado a um computador permitindo, desse modo, a carga e descarga de dados e programas.

\subsubsection{Teclado e Display}

Foram implementados cinco botões, sendo quatro conectados a um registrador 74HCT244 e um ligado diretamente ao sinal INT0 da CPU. Esse último foi pensado como uma forma dos programas serem interrompidos externamente numa aplicação de Stop/Load/Run de uma ROM de carga.

Foi utilizado um display de cristal líquido (LCD) programável ALFACOM de uma linha com dezesseis colunas, modelo LCM 1601 0630. Esse display foi escolhido por ter uma conexão e programação relativamente simples. Todo processo de controle da varredura e da memória interna é feito por comandos de alto nível, enviados ao display, segundo uma tabela fornecida pelo fabricante.

A conexão desse display é feita diretamente ao latch de dados e o endereçamento pelo seletor da forma mais simples possível, pois segue a mesma arquitetura empregada na implementação da conexão dos plugins nas portas de I/O do hardware RoboFácil. 


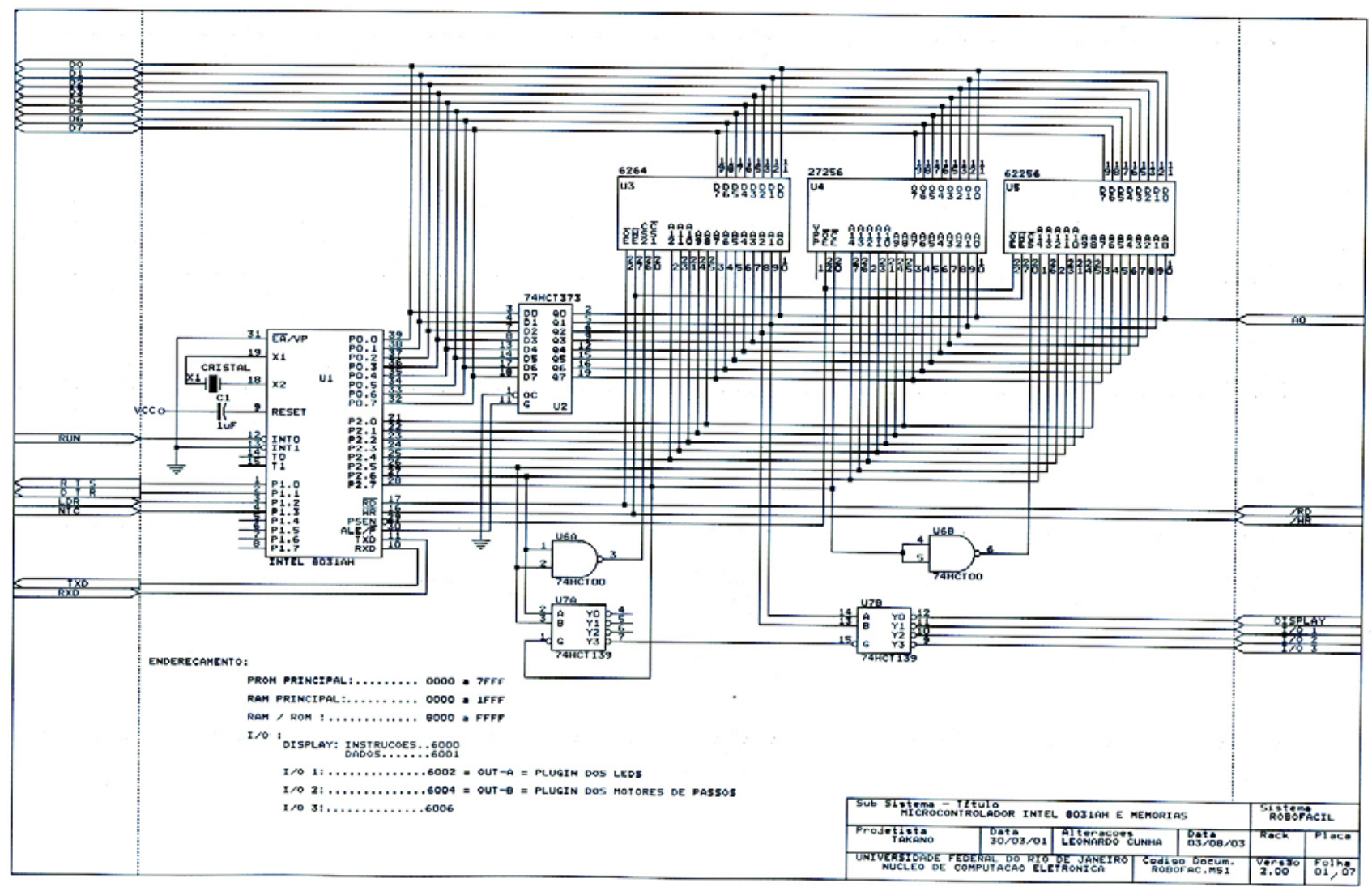

Figura 1: Esquema do circuito eletrônico principal do RoboFácil

\subsubsection{Conversor D/A e A/D}

O hardware provê um conversor digital-analógico (D/A), implementado através de um circuito convencional R-2R, construído sobre um conjunto de resistências. $\mathrm{O}$ valor obtido está na faixa, aproximadamente, de 0 à 4 volts. A entrada do circuito é um registrador 74 HC374 que provê razoável isolamento elétrico.

Para realizar a conversão de sinal analógico para digital (A/D), optamos por não fazer uso de nenhum circuito de conversão rápida, e sim realizar todo controle por comparação de nível. A razão para tal é de fácil entendimento: para medir um nível de entrada analógica, gera-se um valor de tensão no conversor digital-analógico e compara-se o valor obtido com o valor de entrada, através de um comparador analógico construído com amplificadores operacionais LM324 e resistências. Por um processo de busca binária, no máximo em nove comparações, chega-se ao valor da voltagem (valor digital).

Devido à existência de um circuito conversor A/D no hardware RoboFácil torna-se possível no futuro, por exemplo, que o Kit possa ser controlado por comandos de voz.

\subsubsection{Plugins}

Um dos problemas mais complexos encontrados na construção de sistemas robóticos é definir exatamente quais serão as interfaces utilizadas. Colocando poucas interfaces, o sistema fica restrito; se muitas, o sistema fica oneroso e grande. Para solucionar esse impasse foi utilizado no RoboFácil um esquema baseado em plugins. Entendemos que a adoção desses plugins também permite um melhor aproveitamento do RoboFácil como ferramenta educacional, uma vez que flexibiliza sua configuração em diferentes situações de uso.

São dezesseis sinais de saída e dezesseis de entrada, isolados por registradores (74HCT374 e 74HCT244) que podem tolerar correntes relativamente altas protegendo, razoavelmente, o exterior da placa principal do hardwa$r e$. Nessas entradas e saídas, ligadas a dois conjuntos de conectores, se acoplarão os diversos circuitos plugins.

A dificuldade que essa solução introduz é a necessidade de uma programação cuidadosa e dependente das conexões realizadas. A versão atual do hardware RoboFácil conta com quatro plugins para controle de: i) motores de passo; ii) sensor de luminosidade; iii) sensor de temperatura; e iv) LEDs. 


\subsection{Firmware do RoboFácil}

Uma vez definido que o público-alvo desta pesquisa são os estudantes do Ensino Médio, observamos que a dificuldade de utilização do Kit RoboFácil poderia ser elevada, visto que a programação da primeira versão do hardware RoboFácil era realizada apenas utilizando a linguagem Assembly do microcontrolador (MCU) do hardware. Um outro aspecto que poderia comprometer o projeto como um todo era a reduzida velocidade de transferência dos programas desenvolvidos entre o computador e o hardware RoboFácil.

Para contornar os problemas descritos acima e associar outras facilidades ao Kit, optamos pelo desenvolvimento de um novo firmware. Com isso em vista, buscamos no mercado ferramentas que nos permitisse gerar código binário no formato Intel HEX para o MCU do hardware fazendo uso das linguagens Assembly ou C. Dentre as possibilidades existentes, optamos pelo Keil C51 Professional Developers Kit [9], uma vez que esse ambiente de desenvolvimento é constituído pela integração de uma diversidade de ferramentas notadamente úteis aos desenvolvedores de bibliotecas básicas como, por exemplo, compiler, debugger etc.

A criação dessa camada intermediária de software firmware - entre o hardware RoboFácil e os modelos a serem criados pelos usuários garante facilidades na utilização do Kit, pois exime os usuários da necessidade de conhecer questões técnicas aprofundadas sobre esse hardware, ou mesmo, ter que programar esse hardware em linguagem de baixo nível. Desse modo, o controle dos dispositivos eletrônicos/eletromecânicos disponíveis no RoboFácil ficam mais transparentes aos usuários.

A metodologia adotada para dar origem ao novo firmware do RoboFácil foi baseada no paradigma dos sistemas operacionais em camadas. Nessa classe de sistemas uma camada sobrepõe a outra, com a característica de que cada uma possui módulos com um conjunto de funções a serem utilizadas apenas pelos módulos das camadas inferiores. Sendo assim, o firmware foi implementado seguindo semelhante estratégia com o objetivo de isolar funções e facilitar depurações ou incorporações de novas funcionalidades para controle/integração entre todas as soluções computacionais resultantes desta pesquisa.

Demonstramos na Figura 2 as camadas implementadas neste firmware. A camada mais interna (primeira) comunicação serial e envio/recepção de um byte (firmware básico) - compõe o bootstrap do hardware RoboFácil. Essas rotinas foram originalmente escritas na linguagem Assembly durante a primeira fase deste projeto e estão gravadas numa EPROM. Posteriormente, diferentes funcionalidades presentes nessa camada foram reescritas na linguagem $\mathrm{C}$ objetivando criar facilidades de cunho operacional quando de sua integração com as outras camadas. Por sua vez, a segunda camada - bibliotecas básicas de controle de todos os dispositivos eletrônicos - e a terceira camada - interpretador - foram implementadas diretamente na linguagem $\mathrm{C}$.

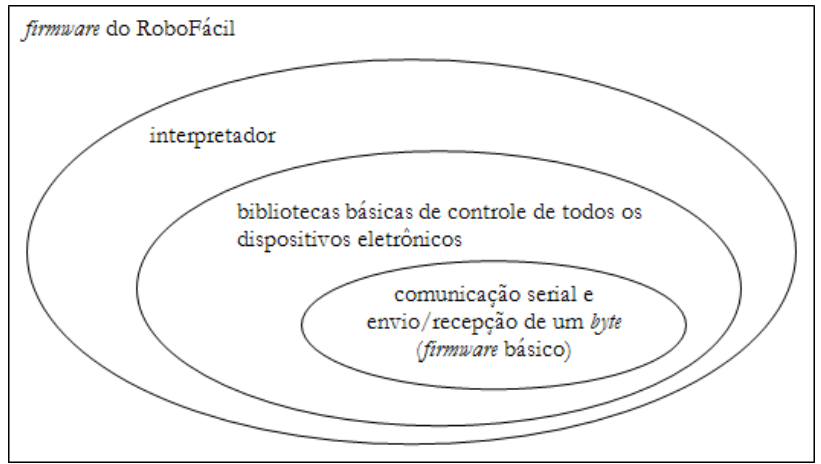

Figura 2: Camadas do firmware do RoboFácil

A camada referente ao interpretador foi idealizada para satisfazer a transcrição do assembly virtual do hardware RoboFácil ${ }^{1}$ em instruções de mais baixo nível compreendidas pelo hardware. Dessa forma, as "tradicionais" palavras - instruções - reservadas presentes na maioria dos assembly serão processadas de forma semelhante tais como: Desviar Para (01h), Carregar Contador (02h), Decrementar Contador (03h), Desviar Se Flag (04h), Desvir Se Não Flag (05h), entre outras.

Salientamos que se o processo de carga do firmware não fosse realizado via software, com a alteração de rotinas haveria a necessidade de regravar a EPROM e essa ROM ainda deveria ser instalada, fisicamente, no hardware RoboFácil. Essas tarefas, para a maioria dos usuários - alunos e professores - que utilizarão o Kit RoboFácil não é intuitivo e nem trivial, necessitando de algumas habilidades específicas e conhecimentos de eletrônica para sua concretização.

Apresentamos na Figura 3, para maior entendimento, os níveis e fluxos dos processos realizados quando da utilização integrada do hardware RoboFácil com sua linguagem de programação visual, ou seja, o software ProgrameFácil.

\footnotetext{
${ }^{1}$ Neste trabalho quando falamos do "assembly virtual do hardware RoboFácil” ou dos "macrocódigos assembly" estamos nos referindo aos códigos hexadecimais definidos neste projeto que representam os pseudocódigos de máquina deste hardware. Esses códigos estão detalhados em [11].
} 


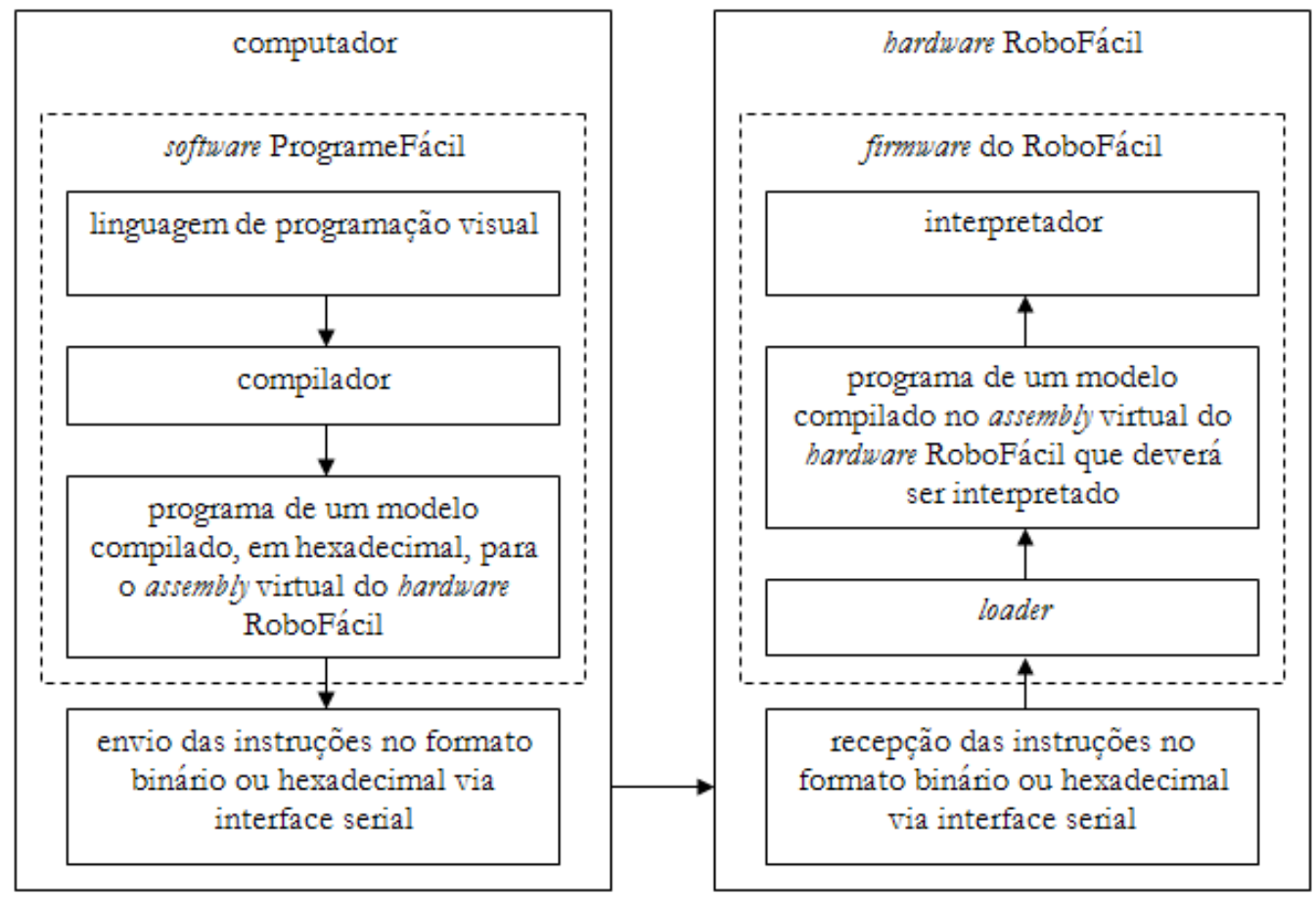

Figura 3: Níveis funcionais entre o hardware RoboFácil e o software ProgrameFácil

\subsubsection{Loader e Interpretador}

Para colocação das instruções do assembly virtual do hardware RoboFácil em formato hexadecimal na RAM de dados do RoboFácil - transferidos via interface serial - foi incorporado ao firmware um novo loader. Assim, após a alocação de um programa na memória, o hardware fica aguardando um comando do usuário - pressionamento de um dos botões do teclado do hardware - para iniciar a execução do programa previamente carregado em sua memória.

A execução de um programa do usuário, instrução a instrução, só é possível a partir da prévia alocação de espaço na RAM de dados. Em seguida, o interpretador executará os macrocódigos assembly enviados via ProgrameFácil, que foram gerados quando da compilação de modelos, permitindo dessa forma que o hardware RoboFácil execute um fluxo lógico de execução.

O assembly virtual do hardware RoboFácil possui dezessete instruções definidas, sendo quatro instruções de 8 bits, sete instruções de 16 bits, quatro instruções de 24 bits e duas instruções de 32 bits. As funções desse conjunto de instruções com seus respectivos parâmetros podem ser consultadas em [11]. Para exercer cada instrução o interpretador segue a seguinte regra: após identificar o código da instrução é verificado se a instrução possui ou não parâmetros. Caso possua, seleciona os parâmetros e executa; não existindo parâmetros, executa a instrução diretamente.
Vale destacar que todas as funcionalidades associadas ao firmware foram agrupadas permitindo, assim, sua utilização de forma simplificada pelo usuário. Na versão atual da solução, a transferência - carregamento - do firmware na memória RAM de programa do hardware RoboFácil é realizada pelo acionamento do botão que representa essa ação na interface do software ProgrameFácil, que será apresentado a seguir. Depois de concluída a transferência, automaticamente, o interpretador é executado, passando a assumir o controle do hardware e aguardando o envio de programas desenvolvidos pelos usuários para serem interpretados neste hardware. É importante ressaltar que, na atual versão do Kit, esse firmware precisa ser transferido apenas uma vez, ou seja, após o hardware RoboFácil ser ligado.

\subsection{Software ProgrameFácil}

A escolha pelo desenvolvimento do software ProgrameFácil foi impulsionada pela necessidade de um ambiente visual - como o RoboLab [12] - que tivesse uma interface de fácil utilização, possibilitando aos usuários da ferramenta programar o comportamento do hardware RoboFácil de forma intuitiva e sem a necessidade de ter que conhecer sua a arquitetura eletrônica.

O ProgrameFácil é uma linguagem de programação visual - do inglês, Visual Programming Language (VPL) - que permite programar dispositivos eletrônicos e eletromecânicos como, por exemplo, lâmpadas, displays, LEDs, motores de passo, e sensores de luminosidade e 
temperatura, fazendo uso das tradicionais construções de programação estruturada, tais como, condicional e repetição. Inicialmente a linguagem foi desenvolvida para controlar o hardware RoboFácil, visto que a primeira versão desse hardware, como comentado anteriormente, era reprogramado apenas através da linguagem Assembly.

As fases de concepção, projeto e implementação da VPL ProgrameFácil levaram sempre em conta a necessidade de criação de um ambiente intuitivo, de forma a torná-lo agradável de usar e eficiente para o controle dos recursos eletrônicos do hardware RoboFácil. Neste sentido, concebemos o ambiente de interação com o usuário como sendo constituído de dois mundos hipotéticos: o primeiro, chamado de $\mathrm{Meu}$ Mundo, onde se especifica a configuração desejada do hardware - motores, sensores, LEDs etc. - e visualiza-se seu funcionamento com a simulação; o segundo, chamado de Meu Programa, onde é permitido explicitar a programação do hardware detalhado no Meu Mundo. Tais mundos são implementados no ProgrameFácil através de duas diferentes janelas, que compõem o ambiente, conforme apresentamos na Figura 4. A adoção de etapas distintas e explícitas para desenhar e executar/simular modelos - mapeados no ambiente em diferentes janelas - tem como objetivo facilitar a averiguação da lógica empregada em cada programa criado pelo usuário.

A fim de garantir maior flexibilidade e qualidade na forma de interação entre a tríade UsuárioProgrameFácil-RoboFácil, incorporamos um compilador a esta VPL e um interpretador ao firmware RoboFácil, conforme descrito na Seção 2.2. O compilador tem como função converter os objetos icônicos que compõem o programa criado pelo usuário em macrocódigos assembly. O interpretador, presente no firmware do RoboFácil permite, por sua vez, converter programas escritos nos macrocódigos assembly, gerados ou não automaticamente pelo ProgrameFácil, em instruções de "mais alto nível" que podem ser executadas no hardware como, por exemplo, acender ou apagar um determinado LED, escrever uma mensagem no display ou movimentar um motor específico.

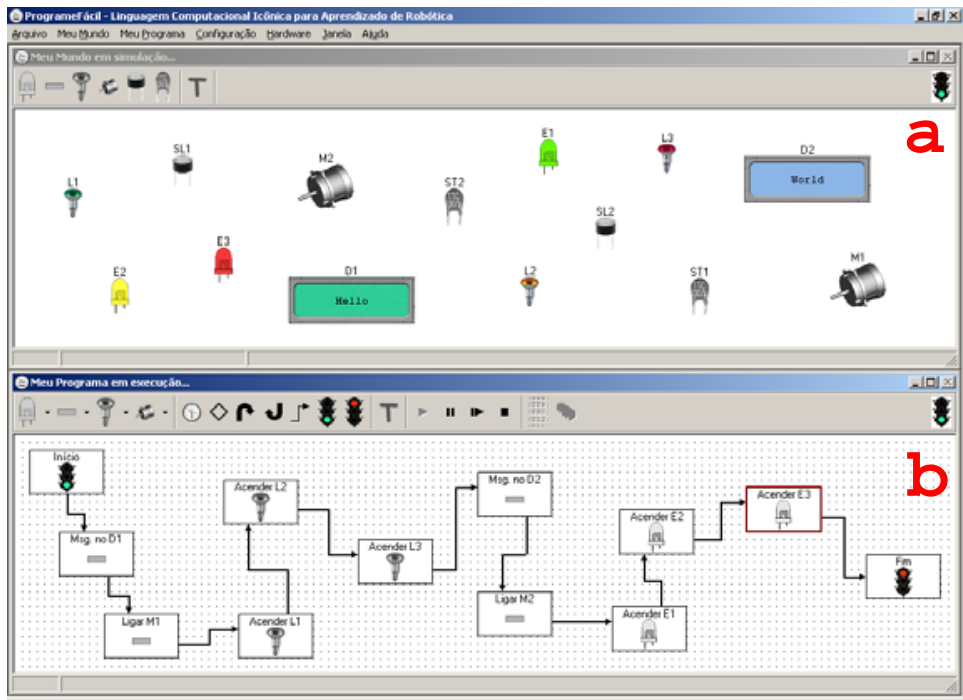

Figura 4: ProgrameFácil apresentando (a) Meu Mundo e (b) Meu Programa

\subsubsection{Modelo de Comportamento e Objetos}

Para um entendimento mais apurado do funcionamento do ProgrameFácil faz-se necessário apresentar a definição do que compreendemos, no contexto desse software, por modelo de comportamento e objetos.

Definimos no ProgrameFácil o termo modelo de comportamento ou, simplesmente, modelo como a configuração de um hardware de robótica, real ou hipotético, com o seu respectivo programa de controle. A materialização dessas ideias no ProgrameFácil foi alcançada através do conjunto de peças/componentes eletrônicos, ou seja, objetos de hardware do Meu Mundo - janela onde é possível especificar a configuração do hardware de um kit de robótica (área a da Figura 4) - e dos objetos de programação do Meu Programa - janela onde é possível definir o programa que irá controlar o funcionamento do hardware de robótica (área b da Figura 4).

O modelo criado pelo usuário no ProgrameFácil poderá retratar ou planejar arquiteturas, casos, atividades, comportamentos, falhas existentes ou não, contribuindo assim para a estruturação do pensamento e posterior simulação acerca dessa modelagem que permitirá infinitas combinações, passíveis de averiguação no que tange à lógica definida. 
Na prática, para se criar um modelo na VPL ProgrameFácil é necessário realizar três etapas distintas: i) especificar os objetos de hardware que serão utilizados no Meu Mundo; ii) incluir no Meu Programa os objetos de hardware e os objetos de programação que serão utilizados; e iii) estabelecer no Meu Programa o fluxo de execução através do objeto Linha de Programação. As formas de manipulação da interface a fim de realizar os passos descritos acima estão detalhados na Ajuda do software.

Um objeto no ProgrameFácil é uma representação gráfica - semelhante a um ícone - que pode ser manipulado tanto no Meu Mundo, como no Meu Programa. Os objetos no ProgrameFácil foram divididos em três categorias para melhor identificar seu propósito: i) objetos de hardware; ii) objetos de programação; e iii) objetos de apoio.

Os objetos de hardware representam dispositivos eletrônicos e foram divididos em duas sub-categorias: i) objetos de hardware de entrada; e ii) objetos de hardware de saída. Os objetos de programação fazem referência às estruturas utilizadas em linguagens de programação. Por sua vez, os objetos de apoio visam unicamente proporcionar facilidades e recursos operacionais aos usuários da ferramenta, tal como, o recurso para incluir comentários textuais nos modelos.

Os objetos de hardware de saída são nomeados igualmente em ambas as janelas. Todavia, possuem características distintas, por exemplo: o objeto LED do $\mathrm{Meu}$

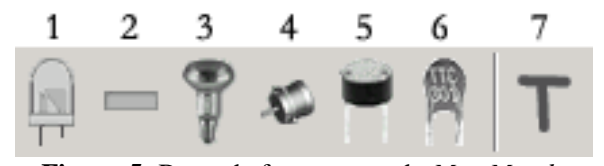

Figura 5: Barra de ferramentas do Meu Mundo

O Meu Programa pode ser definida como o local onde o usuário constrói o programa que irá controlar o funcionamento dos objetos existentes no Meu Mundo. Tal processo se dá através da definição das ações e do encadeamento de estruturas, tais como condicional e repetição, utilizando elementos icônicos para representálas (presentes na barra de ferramentas da janela).
Mundo possui a particularidade de Cor e do Meu Programa a de Acender. Após a inclusão de um objeto de hardware no Meu Mundo é possível alterar as propriedades do mesmo utilizando, por exemplo, o objeto de programação Estrutura de Controle Se. Dessa forma, baseado em decisões lógicas anteriores pode-se, por exemplo, mandar acender ou apagar um determinado LED, escrever uma mensagem no display ou acionar um motor por um certo tempo.

\subsubsection{Meu Mundo e Meu Programa}

O Meu Mundo é a janela onde o usuário poderá especificar o mundo hipotético que representa uma configuração de hardware de um kit de robótica. Os objetos concretos que representam os elementos eletrônicos disponíveis para escolha pelo usuário são apresentados em uma barra de ferramentas dessa janela, com exceção do objeto Comentário que visa unicamente possibilitar a inserção de texto no modelo.

Apresentamos na Figura 5 a barra de ferramentas do Meu Mundo com seus objetos de hardware: 1) LED; 2) Display; 3) Lâmpada; 4) Motor; 5) Sensor de Luminosidade; 6) Sensor de Temperatura; e 7) o recurso de Comentário. Os objetos de hardware passíveis de apresentação no Meu Mundo foram abstraídos da vida real. Portanto, para associá-los a um hardware físico é necessário conhecer suas características físicas e ações permitidas na realidade.
Apresentamos na Figura 6 a barra de ferramentas do Meu Programa com seus objetos de hardware: 1) LED; 2) Display; 3) Lâmpada; e 4) Motor. Também estão disponíveis os objetos de programação: 5) Temporizador; 6) Estrutura de Controle Se; 7) Início de Looping; 8) Fim de Looping; 9) Linha de Programação; 10) Início de Programa; 11) Fim de Programa; e 12) o recurso de Comentário.

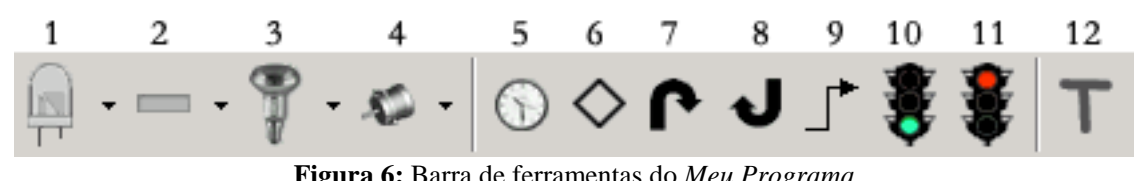

Figura 6: Barra de ferramentas do Meu Programa

A inclusão de objetos de hardware no Meu Programa está atrelada à sua existência no Meu Mundo. Portanto, no Meu Programa, tanto objetos de hardware como objetos de programação podem ser utilizados.
O objetivo é tornar possível a construção de uma lógica de programação entre os elementos presentes, formando assim o que é definido no contexto do ProgrameFácil como o programa do modelo ou, simplesmente, 
programa. Para alcançar esse propósito a linguagem foi construída em conformação com cinco regras de formação:

(i) Cada objeto tem um ou nenhum sucessor na estrutura lógica de programação;

(ii) O objeto Fim de Programa não poderá ter sucessores;

(iii) O objeto Estrutura de Controle Se terá até dois sucessores;

(iv) Cada objeto pode ter um ou mais predecessores na estrutura lógica de programação;

(v) O objeto Início de Programa não poderá ter predecessores.

O programa estabelece a forma de comportamento de um hardware de robótica real ou hipotético e pode ser compreendido como o conjunto de objetos do $\mathrm{Meu}$ Programa com as suas respectivas ações definidas, interligados por um fluxo de execução atemporal, ou seja, um fluxo constituído sem nenhuma referência temporal para execução, estabelecido por meio do emprego do objeto Linha de Programação. O programa inicia com o objeto de programação Início de Programa - semáforo verde e termina no objeto de programação Fim de Programa semáforo vermelho - quando da sua simulação no ambiente ProgrameFácil ou execução no hardware RoboFácil.

\subsubsection{Simulador e Compilador}

O simulador foi incorporado ao software a partir da necessidade de tornar exequível virtualmente o modelo anteriormente criado no computador, antes de realizar a transferência do programa criado para o hardware RoboFácil. Essa funcionalidade, entre outras vantagens, visa proporcionar um momento intermediário de depuração do programa criado. As características de implementação do simulador permitem seu funcionamento de forma automática, após solicitação de início pelo usuário, ou de forma interativa passo-a-passo.

Assim, há dois semáforos agregados ao ambiente sendo um no Meu Mundo e o outro no Meu Programa. Esses semáforos estão posicionados no lado direito de cada janela, funcionam de forma conjunta e representando o mesmo estado. A função desses semáforos é indicar o status do modelo, que pode estar em: desenvolvimento (sinal vermelho em ambas as janelas); pausa (sinal amarelo em ambas as janelas); simulação (sinal verde no $\mathrm{Meu}$
Mundo); e execução (sinal verde no Meu Programa).

No Meu Mundo os objetos do hardware, real ou hipotético, quando em simulação vão alterando suas propriedades baseadas no programa desenvolvido. Conforme a simulação vai prosseguindo, no Meu Programa um ponteiro de execução - retângulo vermelho que contornam os objetos de hardware e de programação do Meu Programa - vai indicando para o usuário o ponto atual de execução do programa. A visualização desse ponteiro é configurável pelo usuário.

Com o intuito de converter o programa desenvolvido na VPL ProgrameFácil nos macrocódigos assembly definidos para o hardware RoboFácil, integramos nesse ambiente um compilador. A tradução é engendrada através da correspondência de macrocódigos assembly para cada objeto de hardware ou de programação presentes no programa. Desse modo, após a compilação, temos um código gerado no assembly virtual do RoboFácil que, ao ser carregado no hardware permite, por exemplo, ligar determinado motor do RoboFácil ou apagar um LED específico do hardware.

Cabe ressaltar que o processo de compilação do programa desenvolvido pelo usuário é realizado apenas com um único click do mouse sobre o botão correspondente a essa funcionalidade disponível na interface do ProgrameFácil, visto que estávamos preocupados com a qualidade da interação do ambiente e conforto na utilização da ferramenta minimizando, por exemplo, pré-requisitos como conhecimentos avançados de programação. Depois de concluída a compilação é apresentada uma janela ao usuário com informações do resultado do processo, ou seja, se a compilação foi realizada com sucesso ou com erros. Caso existam erros, a ferramenta indica possíveis soluções para contorná-los.

\subsubsection{Exemplos}

Alguns exemplos serão apresentados a seguir com o intuito de mostrarmos os recursos implementados e as possibilidades de uso do ambiente.

Apresentamos na Figura 7 um modelo simples construído no ProgrameFácil. Esse primeiro modelo possui um único LED vermelho (E1). Quando esse modelo é executado no ProgrameFácil ou no hardware RoboFácil, E1 irá piscar indefinidamente a cada segundo. 


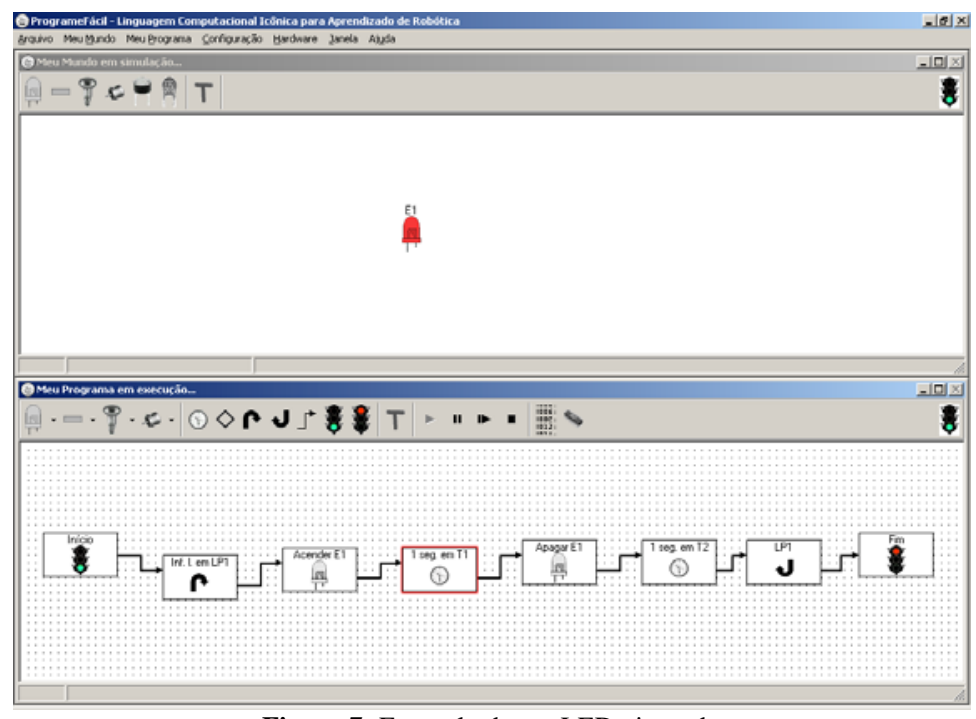

Figura 7: Exemplo de um LED piscando

Na Figura 8 apresentamos um outro exemplo com três lâmpadas - L1 (amarela), L2 (verde) e L3 (vermelha) - e um sensor de luminosidade (SL1). Quando esse modelo for executado no ProgrameFácil ou no hardware RoboFácil, a L1 será ligada e o SL1 será “testado". Se esse sensor identificar a presente de luz no ambiente, a L2 será ligada. Caso contrário, apenas a L3 é ligada. Note que a L1, que está ligada, está perto do SL1, ou seja, esse sensor está identificando presença de luz, portanto, a L2 foi ligada quando esse modelo foi simulado no ProgrameFácil.

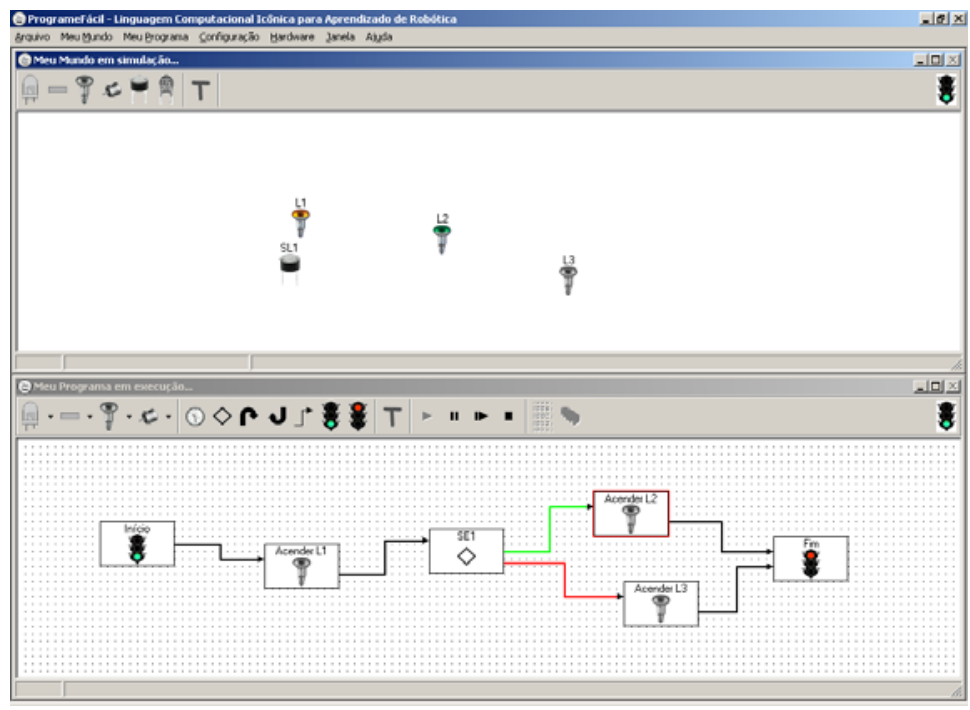

Figura 8: Exemplo com lâmpadas e sensor de luz

Na Figura 9 apresentamos o terceiro exemplo de modelo construído no ProgrameFácil. Esse modelo possui dois LEDs verdes (E1 e E2), um sensor de luminosidade (SL1) e um display alfanumérico com fundo verde fluorescente (D1). O modelo apresentado tem o objetivo de ligar o E2 se o SL1 estiver sob incidência de luz. Quando esse modelo for executado no ProgrameFácil a mensagem "No light" permanecerá escrita no D1 por cinco segundos, enquanto o SL1 não estiver sob incidência de luz (essa foi a condição quando esse modelo foi simulado). 


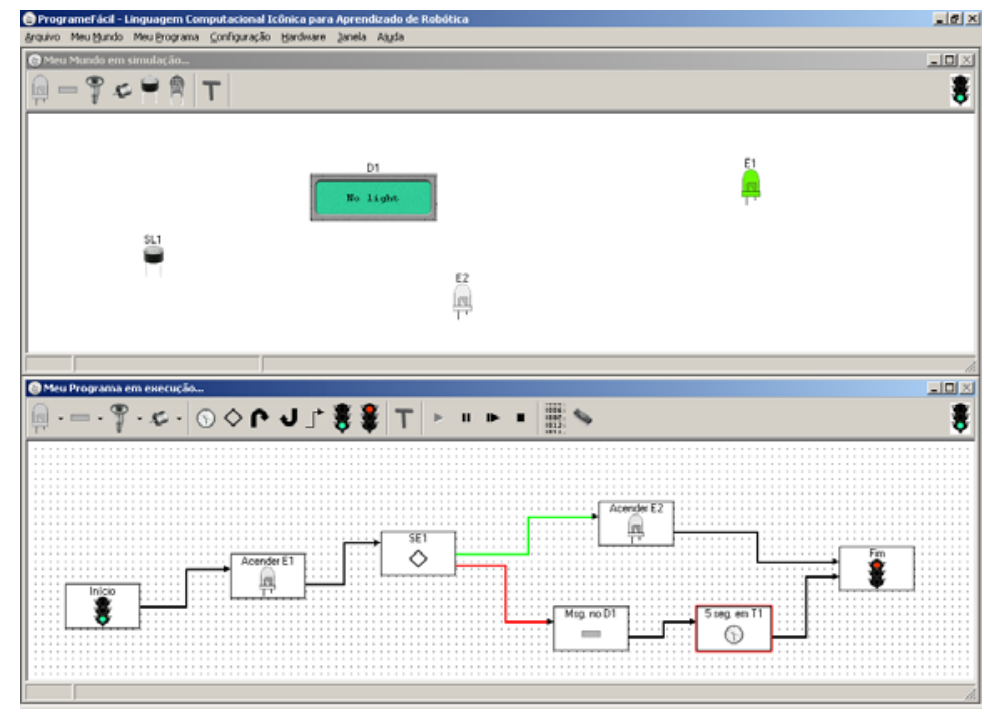

Figura 9: Exemplo com LEDs, sensor de luz e display

O último exemplo apresenta uma situação um pouco mais complexa (Figura 10). Esse modelo possui uma lâmpada amarela (L1), um sensor de temperatura (ST1), dois motores (M1 e M2) e dois displays com fundo azul (D1 e D2). Nesse exemplo, quando o ST1 estiver abaixo do seu ponto de ativação, ou seja, enquanto no modelo a L1 permanecer longe do ST1, o M1 e o M2 serão ligados e esse fato será avisado aos usuários via uma mensagem conjugada escrita no D1 e no D2.

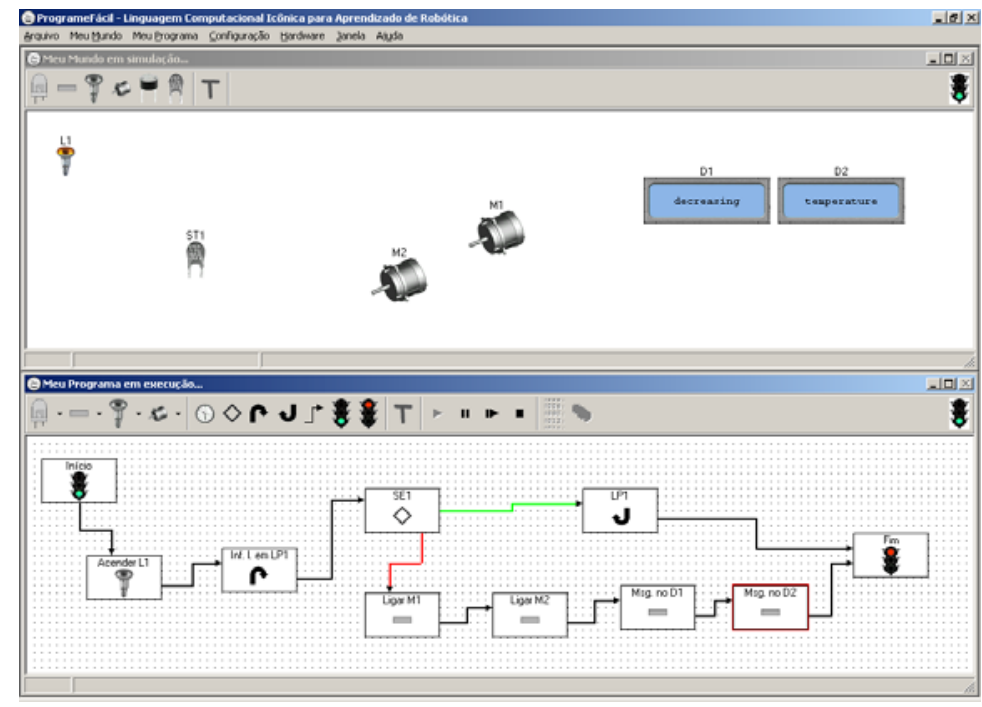

Figura 10: Exemplo com lâmpada, sensor de temperatura, motores e displays

\section{Discussão}

O tema robótica educacional apesar de não ser novo, ainda apresenta relativa escassez de aplicações do gênero no Brasil. Esse fato pode ser corroborado, por exemplo, ao constatarmos o reduzido número de publicações acadêmicas acerca dessa temática, se comparado com outras áreas da Informática na Educação (IE). Entendemos que falta um incentivo dos pesquisadores de IE na realização de trabalhos com foco no desenvolvimento de novos dispositivos de hardware com fins educacionais.

Este trabalho, diferente daqueles que propõem metodologias para uso da robótica educacional em situações reais de ensino - por exemplo, [17] -, possui maior ênfase no desenvolvimento de soluções tecnológicas viáveis a realidade educacional e sócio-financeira brasileira. Assim, no contexto das inovações tecnológicas, cabe nesse momento uma análise comparativa das tecnologias apresentadas neste artigo com outras soluções do gênero que acompanham quatro kits de robótica educacional utiliza- 
dos nas escolas do Brasil.

O software que acompanha o Kit Robótica Fácil [13], diferentemente do ProgrameFácil, é baseado numa interface de linha de comando. O programa que acompanha esse kit apresenta uma quantidade pequena de recursos, apenas possibilitando ligar ou desligar algum dispositivo eletrônico, tais como motor ou lâmpada, e esperar alguma quantidade de tempo. Essa limitação da linguagem pode ser atribuída à arquitetura eletrônica simples desse produto.

O ambiente de programação nativo do Kit Super Robby [19], apesar de ser baseado numa interface visual, possui limitações no desenvolvimento de modelos. Seu ambiente não permite que sejam utilizadas estruturas condicionais para tomada de decisão impossibilitando, por exemplo, que um determinado motor seja ligado caso um sensor de luminosidade identifique a presença de luz. Essa restrição de funcionalidades desse software parece ser reflexo dos poucos recursos de hardware disponibilizados nesse kit.

O Kit GoGo Board [8] não apresenta uma linguagem de programação visual com foco educacional nativo para o seu hardware. Os idealizadores desse hardware disponibilizam na Internet bibliotecas prontas para controlar esse kit através de alguns ambientes comerciais de programação como, por exemplo, Visual C++ e Visual Basic.

O Lego Mindstorms [10] é acompanhado do software RoboLab [12] para controlar seu hardware. Esse é um dos ambientes mais completos para programação visual do kit de robótica da Lego. Entretanto, a versão standard do RoboLab não apresenta algumas funcionalidades que podem ser almejadas por algumas escolas como, por exemplo, a simulação no ambiente de programação.

Nesse contexto, a arquitetura eletrônica adotada pelo hardware RoboFácil o torna diferenciado em relação à maioria dos kits de robótica para fins pedagógicos desenvolvidos no Brasil - por exemplo, [4,5,15] -, pois o mesmo possibilita ter seu comportamento alterado uma infinidade de vezes através de sua reprogramação via uma VPL com foco educacional - ProgrameFácil - não necessitando, portanto, da alteração eletrônica do circuito principal para mudança do comportamento do hardware.

Uma outra particularidade desse hardware é a possibilidade de manter esse equipamento desconectado do computador quando da execução de um programa, previamente desenvolvido e transferido do computador do usuário para o hardware em questão. Tais diferenciais do hardware RoboFácil frente as outras alternativas desenvolvidas no Brasil, entre outras vantagens, proporciona flexibilidade de utilização e aumenta as possibilidades pedagógicas de aplicação deste Kit em diferentes espaços da escola.

Por sua vez, uma importante característica tecnológica do software ProgrameFácil, em relação às outras soluções de software $[8,12,13,19]$ é o recurso de simulação incorporado ao ambiente. Entre outras vantagens, tal característica minimiza a necessidade de se possuir um hardware para cada grupo de alunos contribuindo, assim, para a redução de custos na montagem de laboratórios de robótica. Entretanto, é importante ressaltar que se deve ter um número de kits suficientes para que todos os alunos participem do processo de construção/montagem e controle do hardware de robótica educacional viabilizando, de fato, o "fazer-aprendendo" e o colocar a "mão-namassa”.

Vale relembrar que a VPL ProgrameFácil foi originalmente projetada para utilização com o Kit RoboFácil. Entretanto, dada as suas características de implementação é possível integrá-la a diferentes projetos de hardware de robótica, afim de empregá-la em situações de sala de aula voltadas ao Ensino Técnico/Profissionalizante, Superior e de Pós-Graduação.

De forma sintética, apontamos os seguintes itens como principais diferenciais do Kit RoboFácil: i) hardware modular e escalonável com a adoção dos plugins eletrônicos; ii) hardware reprogramável via software; iii) VPL para kits de robótica com foco educacional; iv) sistema aberto; v) projeto e implementação totalmente nacional; e vi) boa relação custo/benefício.

\section{Considerações Finais}

A relevância desta pesquisa está na fusão de conhecimentos de distintas áreas das engenharias e ciência da computação que, integrados, proporcionaram a implementação de um kit de robótica com fins educacionais composto do hardware RoboFácil e do software ProgrameFácil. O kit de robótica educacional RoboFácil e suas características, como visto neste artigo, o fazem diferente das soluções existentes no mercado brasileiro. O Kit visa atender a um público carente de alternativas nessa área como, por exemplo, os alunos do Ensino Médio. Não descartando, todavia, outros segmentos do Ensino.

Conjeturamos, num contexto sócio-técnico, que as soluções aqui apresentadas poderão ser utilizadas como base para novos desenvolvimentos, permitindo-nos também vislumbrar uma maior utilização da robótica com fins pedagógicos por segmentos da sociedade ainda não atingidos com essa tecnologia. Visualizamos a utilização deste Kit integrado às atividades curriculares de disciplinas das Engenharias, Ciência da Computação, Física e Matemática, visto que metodologias didáticas que privi- 
legiem o uso de artefatos tecnológicos em situações reais de ensino abrem oportunidades aos discentes de relacionarem aspectos conceituais com situações do dia-a-dia.

Algumas simulações de uso em contexto educacional realizadas com alunos de Pós-Graduação em Informática, demonstraram o potencial de aplicação das soluções aqui descritas. Tais testes permitiram ao nosso grupo de pesquisa obter uma visão mais prática da utilização dessas tecnologias em ambientes de ensino, nos dando um feedback de algumas melhorias a serem implementadas. Como estudo futuro propomos a realização de um trabalho para viabilizar que outros hardware de robótica - por exemplo, Arduino [2] - possam ser programados e, até mesmo, simulados via ProgrameFácil.

\section{Referências}

[1] A. C. Alves, P. Blikstein, R. D. Lopes. Robótica na Periferia? Uso de Tecnologias Digitais na Rede Pública de São Paulo como Ferramentas de Expressão e Inclusão. In Anais do XXV Congresso da Sociedade Brasileira de Computação, São Leopoldo, páginas 2594-2602, 2005.

[2] Arduino. http://www.arduino.cc, Dez. 2010.

[3] M. T. Chella. Ambiente de Robótica para Aplicações Educacionais com SuperLogo. Dissertação de Mestrado, Universidade Estadual de Campinas, Outubro 2002.

[4] S. M. S. Cruz, L. R. Sasahara, K. Sasahara et al. Hajime: O Relato de um Experimento em Robótica Educacional de Baixo Custo. In Anais do XVIII Simpósio Brasileiro de Informática na Educação. São Paulo, páginas 176-179, 2007.

[5] M. E. J. K. Cruz, B. Lux, W. Haetinger et al. Formação Prática do Licenciando em Computação para Trabalho com Robótica Educativa. In Anais do XVIII Simpósio Brasileiro de Informática na Educação. São Paulo, páginas 422-431, 2007.

[6] J. V. V. d'Abreu, L. M. G. Gonçalves, M. F. Garcia et al. Uma Abordagem PráticoPedagógica para o Ensino de Robótica em Ciência e Engenharia de Computação. In Anais do XIII Simpósio Brasileiro de Informática na Educação, Porto Alegre, páginas 428-439, 2002.

[7] J. V. V. d'Abreu. Desenvolvimento de Ambientes de Aprendizagem Baseados no Uso de Dispositivos Robóticos. In Anais do X Simpósio Brasileiro de Informática na Educação, Curiti- ba, páginas 9-16, 1999.

[8] Gogo Board. http://www.gogoboard.org, Dez. 2010.

[9] Keil C51 Professional Developers Kit. http://www.keil.com/c51, Dez. 2010.

[10] Lego Mindstorms. http://mindstorms.lego.com, Dez. 2010.

[11] L. C. Miranda. RoboFácil: Especificação e Implementação de Artefatos de Hardware e Software de Baixo Custo para um Kit de Robótica Educacional. Dissertação de Mestrado, Universidade Federal do Rio de Janeiro, Abril 2006.

[12] RoboLab Software. http://www.lego.com/eng/education/mindstorms/ home.asp?pagename=robolab.

[13] Robótica Fácil. Manual do Kit Robótica Fácil, 2003.

[14] C. F. Santos, C. S. Menezes. A Aprendizagem da Física no Ensino Fundamental em um Ambiente de Robótica Educacional. In Anais do XXV Congresso da Sociedade Brasileira de Computação, São Leopoldo, páginas 2746-2753, 2005.

[15] L. R. Sasahara, S. M. S. Cruz. Hajime - Uma Nova Abordagem em Robótica Educacional. In Anais do XXVII Congresso da Sociedade Brasileira de Computação, Rio de Janeiro, páginas 459-461, 2007.

[16] C. Schons, E. Primaz, G. A. P. Wirth. Introdução a Robótica Educativa na Instituição Escolar para Alunos do Ensino Fundamental da Disciplina de Língua Espanhola através das Novas Tecnologias de Aprendizagem. In Anais do I Workshop de Computação da Região Sul, 2004.

[17] A. F. Silva. RoboEduc: Uma Metodologia de Aprendizado com Robótica Educacional. Tese de Doutorado, Universidade Federal do Rio Grande do Norte, Março 2009.

[18] H. H. Steffen. Robótica Pedagógica na Educação: Um Recurso de Comunicação, Regulagem e Cognição. Dissertação de Mestrado, Universidade de São Paulo, 2002.

[19] Super Robby. http://www.arsconsult.com.br/produtos/srobby.

[20] S. R. Zilli. A Robótica Educacional no Ensino Fundamental: Perspectivas e Prática. Dissertação de Mestrado, Universidade Federal de Santa Catarina, Outubro 2004. 